\title{
ELECTRONIC ENERGIES OF INTERACTION OF POINT DEFECTS WITH INTERFACES AND GRAIN BOUNDARIES
}

\author{
J.O. Vasseur, P.A. Deymier*, A. Akjouj, L. Dobrzynski \\ AND B. DJaFari-RouhanI \\ Equipe Internationale de Dynamique des Interfaces \\ Laboratoire de Dynamique et Structure des Matériaux Moléculaires, \\ U.R.A. CNRS $n^{\circ} 801$ - UFR de Physique, Université de Lille I \\ 59655 Villeneuve d'Ascq, Cédex, France
}

(Received May 21, 1991)

The electronic energies of interaction of a point defect with interfaces and grain boundaries are calculated, as a function of the distance $x_{03}$ between the point defect and the corresponding planar defect. These interaction energies are shown to have the same type of oscillations as the corresponding local electronic densities. Therefore the electronic interactions more significantly affect the energy of segregation of point defects towards interfaces when $x_{03}$ is in the near vicinity of these interfaces, while at large distances the contribution of elastic interactions can dominate.

PACS numbers: 71.20.-b, 73.40.-c, 61.70.Yq, 68.35.Dv

Grain boundary segregation is an area of intense interest since solute atoms concentrating at grain boundaries can alter drastically the properties of bulk polycrystalline materials. Elastic and electronic interactions are the major contributions to equilibrium segregation of impurities to grain boundaries in simple metals.

In the previous publication [1], analytic expressions were reported for the elastic energy of interaction of a point defect with a planar defect. The excess elastic energy of the point defect in the vicinity of the planar defect was found to be short range and to vary as $1 / d^{4}$ where $d$ is the distance from the planar defect. This energy was expected to be a leading contribution to segregation.

The electronic energy of interaction of a solute atom with a grain boundary is believed to offer an insufficient driving force for segregation. Friedel [2] presented a variation of the electronic energy exhibiting damped oscillations as a function of the distance. Atomistic calculations based on pair potentials or on tight-binding models have been performed to determine the excess energy of solute atoms at

*Permanent address: Department of Materials Science and Engineering, University of Arizona, Tucson, AZ 85721, USA. 
different sites in the vicinity of surfaces $[3,4]$ or near a grain boundary [5]. The density functional formalism was also applied to obtain the electronic density near a planar defect and the energy of a grain boundary [6].

The purpose of this paper is to obtain within a simple but general model the excess energy of a point defect near an interface between two metals $M_{1}$ and $M_{2}$, or near a planar defect modelled as a thin slab of metal $\mathrm{M}_{2}$ sandwiched between two semi-infinite perfect crystals of metal $M_{1}$. This energy will be expressed as a simple integral which is easy to evaluate. Furthemore the Green function method used in this work can easily be extended to more complicated heterostructures. The calculations are conducted in the nearly free electron model, with step barriers at the interfaces.

The perturbation potential due to solute atom located at $r_{0}$ is assumed to be a point-like potential

$$
V(r)=A_{0} \delta\left(r-r_{0}\right)
$$

where $\delta(r)$ is the usual delta function and $A_{0}$ defines the strength of the perturbation.

The correction to a non-degenerated electronic energy level of the system due to this potential is written, to the first order in the perturbation $V(r)$, in the form

$$
\varepsilon\left(r_{0}, E\right)=\langle\Psi|V| \Psi\rangle
$$

where $|\Psi\rangle$ is an eigenvector of the unperturbed system associated with the energy $E$. Introducing Eq. (1) in (2) gives

$$
\varepsilon\left(r_{0}, E\right)=A_{0} n_{0}\left(r_{0}, E\right),
$$

where $n_{0}\left(r_{0}, E\right)$ is the contribution of the electrons with energy $E$ to the electronic density at location $r_{0}$ in the unperturbed interface or sandwich system.

Furthermore, the electronic density $n_{0}\left(r_{0}, E\right)$ can be advantageously determined from the imaginary part of the Green function $g\left(r_{0}, r_{0}, E\right)$ of the unperturbed system by the relation

$$
n\left(r_{0}, E\right)=-\frac{1}{\pi} \operatorname{Im} g\left(r_{0}, r_{0}, E\right) .
$$

Then the total energy of the point defect in the $M_{1}-M_{2}$ or in the $M_{1}-M_{2}-M_{1}$ composite system is obtained at $0 \mathrm{~K}$ by summing $\varepsilon\left(r_{0}, E\right)$ over all possible states inside the Fermi surface

$$
\varepsilon\left(r_{0}\right)=A_{0} n\left(r_{0}\right),
$$

where $n\left(r_{0}\right)$ is the electronic density at location $r_{0}$

$$
n\left(r_{0}\right)=-\frac{1}{\pi} \operatorname{Im} \int^{E_{\mathrm{F}}} \mathrm{d} E g\left(r_{0}, r_{0}, E\right) .
$$

The calculation of the Green function is first performed in the two-dimensional space parallel to the interfaces by using the interface response theory of Ref. [7]. This means that we express the elements $g\left(k_{\|} ; x_{03} ; x_{03}^{\prime} ; E\right)$ of the Green function, 
where $k_{\|}$is the wave vector parallel to the interfaces and $x_{03}$ is the direction perpendicular to them. These expressions are given in the Appendix 1. Then

$$
n\left(r_{0}\right)=-\frac{1}{\pi} \operatorname{Im} \int^{E_{\mathrm{F}}} \mathrm{d} E \int \frac{\mathrm{d}^{2} k_{\|}}{(2 \pi)^{2}} g\left(k_{\|} ; x_{03} ; x_{03} ; E\right) .
$$

In our calculation the metals $M_{1}$ and $M_{2}$ involved in the $M_{1}-M_{2}$ interface system or in the $\mathrm{M}_{1}-\mathrm{M}_{2}-\mathrm{M}_{1}$ planar defect are characterized by their ground energies $E_{1}, E_{2}$ and their effective masses $m_{1}, m_{2}$. In the nearly free electron approximation these parameters are related to the bulk electronic density $n_{i}$ in metal $i$ by the relation

$$
E_{\mathrm{F}}-E_{i}=\frac{\hbar^{2}}{2 m_{i}}\left(\frac{3 n_{i}}{8 \pi}\right)^{2 / 3} \quad i=1,2 .
$$

The Fermi levels of both metals are aligned in the composite system at the energy $E_{\mathrm{F}}$. We also introduce the quantities

with

$$
\alpha_{i}^{2}=k_{\|}^{2}-\frac{\left(E-E_{i}\right)}{B_{i}}
$$

$$
B_{i}=\frac{2 m_{i}}{\hbar^{2}}
$$

For the sake of simplicity, we chose the effective mass of the electron to be the same in both media $\mathrm{M}_{1}$ and $\mathrm{M}_{2}\left(B_{1}=B_{2}=B\right)$.

Finally the geometries of the systems are defined as follows. In the interface problem, the metal $\mathrm{M}_{1}\left(\mathrm{M}_{2}\right)$ fills the negative (positive) $x_{3}$ half-space. In the planar defect system, the slab $M_{2}$ fills the region $-X<x_{3}<+X$, where $x_{3}= \pm X$ define the locations of the interfaces.

Let us assume that $E_{1}$ is below $E_{2}$ which means that the electron density is smaller in material $\mathrm{M}_{2}$ than in material $\mathrm{M}_{1}$. This is expected to be the case in high angle grain boundaries which are dilated regions showing lower atomic densities than in the bulk. The multiple integral in Eq. (4c) can be simplified by changing the variables $u^{2}=\left(E-E_{i}\right) / B_{1}-k_{\|}^{2}$ and $k^{2}=2 m\left(E-E_{1}\right) / \hbar^{2}$ and by inverting the order of integration over $u$ and $k$. After some algebra, we obtain $n\left(r_{0}\right)$ as a simple integral of the form

$$
n\left(x_{03}\right)=\frac{1}{2 \pi^{2}} \int_{0}^{k_{\mathrm{F}}}\left(k_{\mathrm{F}}^{2}-u^{2}\right) \operatorname{Im} g\left(u, x_{03}\right) u \mathrm{~d} u .
$$

The expression of $g\left(u, x_{03}\right)$ which is dependent upon the location $x_{03}$ of the impurity is given in Appendix 2.

As the first application of this calculation, Fig. 1 presents the variation of $n\left(x_{03}\right)$ near a bimetallic interface $\mathrm{M}_{1}-\mathrm{M}_{2}$. The bulk charge density in metals $\mathrm{M}_{1}$ and $\mathrm{M}_{2}$ are chosen in order to simulate an $\mathrm{Al}-\mathrm{Ag}$ system. Apart from a rapid variation near the interface, the charge density shows decaying oscillations with period $\pi / k_{\mathrm{F}}$ before reaching the bulk value on each side of the interface. In a self-consistent calculation as in Ref. [8], the oscillations are actually less pronounced than those in Fig. 1. 


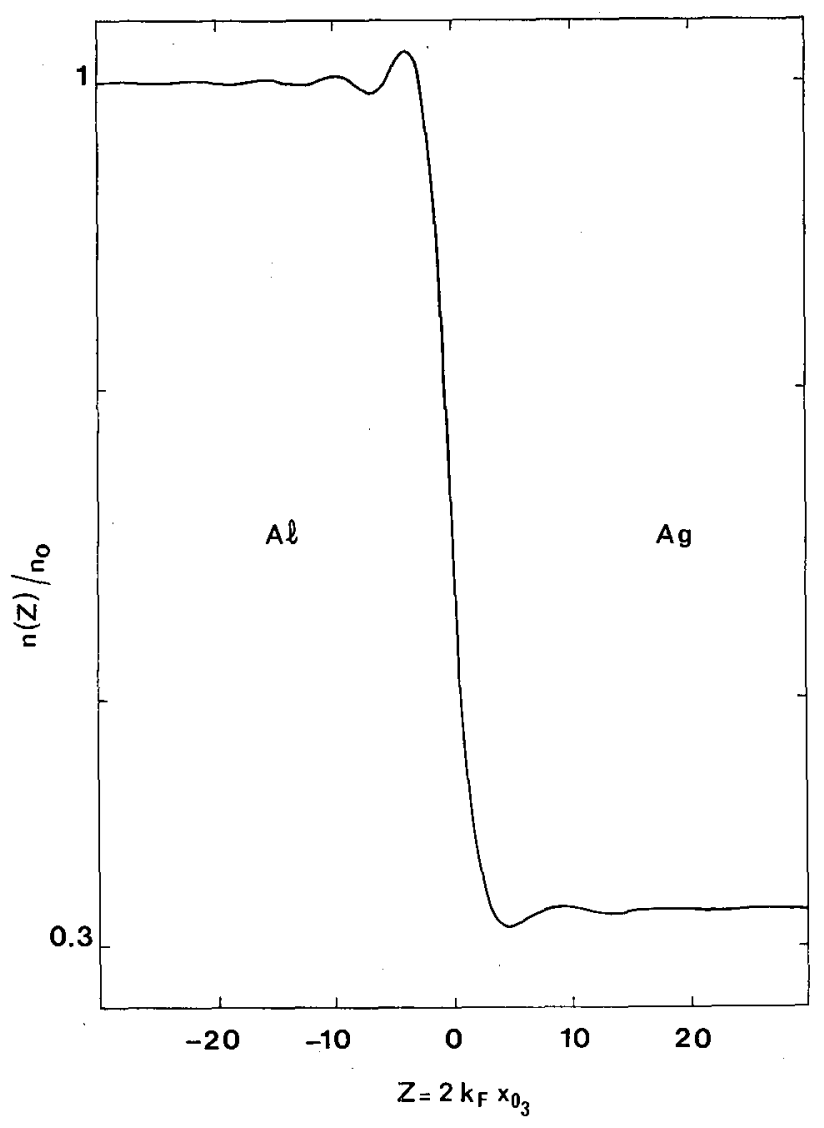

Fig. 1. Electronic density near a $\mathrm{Al}-\mathrm{Ag}$ interface, normalised to the bulk electronic density in Al.

In Fig. 2, we present the behaviour of the electronic density in a Al crystal containing a planar defect. The electronic density inside the planar defect is chosen to be $20 \%$ smaller than in the bulk crystal in order to simulate a smaller atomic density inside a grain boundary. The curves in Fig. 2 refer to different thicknesses $2 X$ of the planar defect.

Again one can notice a rapid variation of the electronic density in the near vicinity of the interfaces. Inside a relatively thick planar defect $n\left(x_{03}\right)$ shows decaying oscillations before reaching a limiting value; in contrast, in a very thin planar defect $n\left(x_{03}\right)$ behaves rather parabolically with a minimum at $x_{03}=0$ which is higher than in the thick grain boundary case.

Outside the grain boundary, the behaviour of the electronic density is qualitatively similar to that in the bimetallic system, except for a very thin planar defect where the charge density is influenced by the presence of both interfaces.

As a conclusion, we can notice that at large distances from the planar defect, 


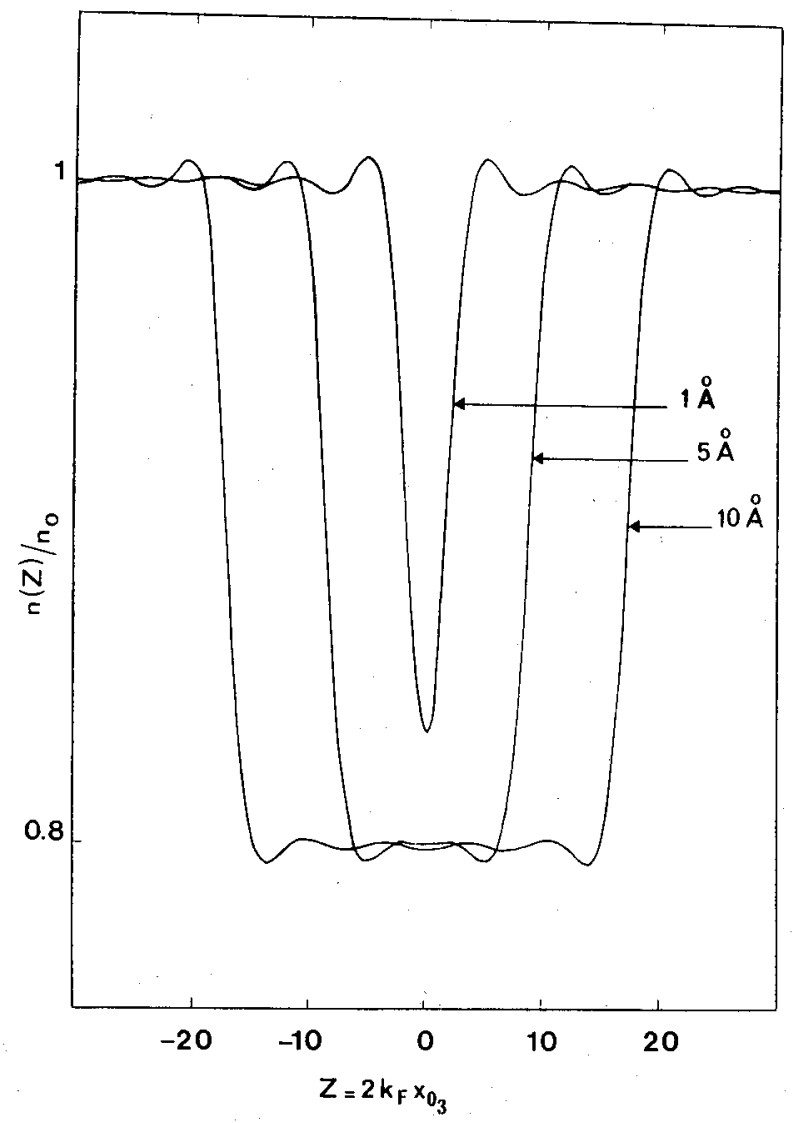

Fig. 2. Electronic density near a planar defect in an Al crystal, for three different thicknesses $(1,5$ and $10 \AA)$ of the planar defect. The density is normalised to the bulk density of Al. The atomic density in the grain boundary is assumed to be $20 \%$ lower than in the bulk crystal.

the electronic energy shows an attenuated sinusoidal variation which gives rise to an oscillating driving force changing sign every fraction of an interatomic distance; therefore this mechanism does not provide a sufficient driving force for segregation of impurities. In contrast, at very short distances, that is within a few atomic radii from the grain boundary core, the electronic energy may represent a significant contribution to the energy of segregation. This is in accordance with measurements of the extent of the equilibrium grain boundary segregation which indicate the limitation of the segregation phenomenon to a few atomic layers at the grain boundary core [9].

Finally let us mention that although only qualitative results can be expected from our simple approach, the method of calculation can easily be extended to other heterostructures of more complex geometries. 


\section{Appendix 1}

Here we report the Green function $g\left(k_{\|}, x_{3}, x_{3}^{\prime} ; E\right)$ for a $\mathrm{M}_{1}-\mathrm{M}_{2}$ interface system and a $\mathrm{M}_{1}-\mathrm{M}_{2}-\mathrm{M}_{1}$ sandwich system in the approximation of the nearly free electrons.

1) $M_{1}-M_{2}$ interface system

- In the medium $M_{1}\left(x_{3}, x_{3} \leq 0\right)$

$$
g\left(k_{\|}, x_{3}, x_{3}^{\prime} ; E\right)=-\frac{1}{2 \alpha_{1} B_{1}}\left[\mathrm{e}^{-\alpha_{1}\left|x_{3}-x_{3}^{\prime}\right|}+\frac{\alpha_{1} B_{1}-\alpha_{2} B_{2}}{\alpha_{1} B_{1}+\alpha_{2} B_{2}} \mathrm{e}^{\alpha_{1}\left(x_{3}+x_{3}^{\prime}\right)}\right] ;
$$

- In the medium $\mathrm{M}_{2}\left(x_{3}, x_{3}^{\prime} \geq 0\right)$

$$
g\left(k_{\|} ; x_{3}, x_{3}^{\prime}, E\right)=-\frac{1}{2 \alpha_{2} B_{2}}\left[\mathrm{e}^{-\alpha_{2}\left|x_{3}-x_{3}^{\prime}\right|}-\frac{\alpha_{1} B_{1}-\alpha_{2} B_{2}}{\alpha_{1} B_{1}+\alpha_{2} B_{2}} \mathrm{e}^{-\alpha_{2}\left(x_{3}+x_{3}^{\prime}\right)}\right] .
$$

2) $\mathrm{M}_{1}-\mathrm{M}_{2}-\mathrm{M}_{1}$ sandwich system:

- In the medium $\mathrm{M}_{1}$ with $x_{3}, x_{3}^{\prime} \leq-X$

$$
\begin{aligned}
& g\left(k_{\|} ; x_{3}, x_{3}^{\prime}, E\right)=-\frac{\mathrm{e}^{-\alpha_{1}\left|x_{3}-x_{3}^{\prime}\right|}}{2 \alpha_{1} B_{1}}+\frac{\mathrm{e}^{\alpha_{1}\left(x_{3}+x_{3}^{\prime}+2 X\right)}}{2 \alpha_{1} B_{1}} \\
& -\frac{\alpha_{1} B_{1} \operatorname{th}\left(2 \alpha_{2} X\right)+\alpha_{2} B_{2}}{W\left(k_{\|}, E\right)} \mathrm{e}^{\alpha_{1}\left(x_{3}+x_{3}^{\prime}+2 X\right)} ;
\end{aligned}
$$

- In the medium $\mathrm{M}_{2}\left(-X \leq x_{3}, x_{3}^{\prime} \leq+X\right)$

$$
\begin{aligned}
& g\left(k_{\|} ; x_{3}, x_{3}^{\prime}, E\right)=-\frac{\mathrm{e}^{-\alpha_{2}\left|x_{3}-x_{3}^{\prime}\right|}}{2 \alpha_{2} B_{2}}+\frac{1}{2 \alpha_{2} B_{2} \operatorname{sh}\left(2 \alpha_{2} X\right)}\left\{\operatorname { c h } \left[\alpha_{2}\left(x_{3}+x_{3}^{\prime}\right)\right.\right. \\
& \left.-\mathrm{e}^{-2 \alpha_{2} X} \operatorname{ch}\left[\alpha_{2}\left(x_{3}-x_{3}^{\prime}\right)\right]\right\}-\frac{1}{2 W\left(k_{\|}, E\right) \operatorname{sh}^{2}\left(2 \alpha_{2} X\right)}\left\{\left[\alpha_{1} B_{1} \operatorname{th}\left(2 \alpha_{2} X\right)+\alpha_{2} B_{2}\right]\right. \\
& \times\left[\operatorname{ch}\left[\alpha_{2}\left(x_{3}+x_{3}^{\prime}-2 X\right)\right]+\operatorname{ch}\left[\alpha_{2}\left(x_{3}+x_{3}^{\prime}+2 X\right)\right]-2 \operatorname{ch}\left[\alpha_{2}\left(x_{3}-x_{3}^{\prime}\right)\right]\right] \\
& +\frac{\alpha_{2} B_{2}}{\operatorname{ch}\left(2 \alpha_{2} X\right)}\left[\operatorname{ch}\left[\alpha_{2}\left(x_{3}-x_{3}^{\prime}+2 X\right)\right]\right. \\
& \left.\left.+\operatorname{ch}\left[\alpha_{2}\left(x_{3}-x_{3}^{\prime}-2 X\right)\right]-2 \operatorname{ch}\left[\alpha_{2}\left(x_{3}+x_{3}^{\prime}\right)\right]\right]\right\}
\end{aligned}
$$

- In the medium $M_{1}$ with $x_{3}, x_{3}^{\prime} \geq+X$

$$
\begin{aligned}
& g\left(k_{\|} ; x_{3}, x_{3}^{\prime}, E\right)=-\frac{\mathrm{e}^{-\alpha_{1}\left|x_{3}-x_{3}^{\prime}\right|}}{2 \alpha_{1} B_{1}}+\frac{\mathrm{e}^{-\alpha_{1}\left(x_{3}+x_{3}^{\prime}-2 X\right)}}{2 \alpha_{1} B_{1}} \\
& -\frac{\alpha_{1} B_{1} \operatorname{th}\left(2 \alpha_{2} X\right)+\alpha_{2} B_{2}}{W\left(k_{\|}, E\right)} \mathrm{e}^{-\alpha_{1}\left(x_{3}+x_{3}^{\prime}-2 X\right) .} .
\end{aligned}
$$

In these expressions the denominator $W\left(k_{\|}, E\right)$ is given by $W\left(k_{\|}, E\right)=\left(\alpha_{1}^{2} B_{1}^{2}+\alpha_{2}^{2} B_{2}^{2}\right) \operatorname{th}\left(2 \alpha_{2} X\right)+2 \alpha_{1} B_{1} \alpha_{2} B_{2}$. 


\section{Appendix 2}

In this appendix, we give the expressions of $g\left(u, x_{03}\right)$ appearing in Eq. (7) according to the location $x_{03}$ of the impurity.

1) $M_{1}-M_{2}$ interface system:

For $x_{03} \leq 0$

$$
g\left(u, x_{03}\right)=-\frac{\mathrm{i}}{2 u B}\left(1+\frac{\mathrm{i} u+\alpha_{2}}{\mathrm{i} u-\alpha_{2}} \mathrm{e}^{-2 \mathrm{i} u x_{03}}\right)
$$

where

$$
\alpha_{2}= \begin{cases}\sqrt{k_{0}^{2}-u^{2}} & \text { if } u \leq k_{0} \\ -\mathrm{i} \sqrt{u^{2}-k_{0}^{2}} & \text { if } u \geq k_{0}\end{cases}
$$

and $k_{0}=\sqrt{\left(E_{2}-E_{1}\right) / B}$.

For $x_{03} \geq 0$

$$
g\left(u, x_{03}\right)=-\frac{1}{2 \alpha_{2} B}\left(1+\frac{\mathrm{i} u+\alpha_{2}}{\mathrm{i} u-\alpha_{2}} \mathrm{e}^{-2 \alpha_{2} x_{03}}\right) .
$$

2) $\mathrm{M}_{1}-\mathrm{M}_{2}-\mathrm{M}_{1}$ sandwich system

For $x_{03} \leq-X$

$$
g\left(u, x_{03}\right)=\frac{-\mathrm{i}\left(1-\mathrm{e}^{-2 \mathrm{i} u\left(x_{03}+X\right)}\right)}{2 u B}-\frac{\left(\alpha_{2}-\mathrm{i} u \operatorname{th}\left(2 \alpha_{2} X\right)\right) \mathrm{e}^{-2 \mathrm{i} u\left(x_{03}+X\right)}}{B\left[\left(\alpha_{2}^{2}-u^{2}\right) \operatorname{th}\left(2 \alpha_{2} X\right)-2 \mathrm{i} u \alpha_{2}\right]} .
$$

For $-X \leq x_{03} \leq+X$

$$
\begin{aligned}
& g\left(u, x_{03}\right)=-\frac{1}{2 \alpha_{2} B}+\frac{1}{2 \alpha_{2} B \operatorname{sh}\left(2 \alpha_{2} X\right)}\left[\operatorname{ch}\left(2 \alpha_{2} x_{03}\right)-\mathrm{e}^{-2 \alpha_{2} X}\right] \\
& -\frac{1}{B \operatorname{sh}^{2}\left(2 \alpha_{2} X\right)\left[\left(\alpha_{2}^{2}-u^{2}\right) \operatorname{th}\left(2 \alpha_{2} X\right)-2 \mathrm{i} u \alpha_{2}\right]} \\
& \left.\times\left\{\left(-\mathrm{i} u \operatorname{th}\left(2 \alpha_{2} X\right)+\alpha_{2}\right)\left[\operatorname{ch}\left(2 \alpha_{2} x_{03}\right) \operatorname{ch}\left(2 \alpha_{2} X\right)-1\right]\right)+\alpha_{2}\left[1-\frac{\operatorname{ch}\left(2 \alpha_{2} x_{03}\right)}{\operatorname{ch}\left(2 \alpha_{2} X\right)}\right]\right\} .
\end{aligned}
$$

For $x_{03} \geq+X$

$$
g\left(u, x_{03}\right)=\frac{-\mathrm{i}\left[1-\mathrm{e}^{2 \mathrm{i} u\left(x_{03}-X\right)}\right]}{2 u B}-\frac{\left[\alpha_{2}-\mathrm{i} u \operatorname{th}\left(2 \alpha_{2} X\right)\right] \mathrm{e}^{2 \mathrm{i} u\left(x_{03}-X\right)}}{B\left[\left(\alpha_{2}^{2}-u^{2}\right) \operatorname{th}\left(2 \alpha_{2} X\right)-2 \mathrm{i} u \alpha_{2}\right]} .
$$

\section{References}

[1] P. Deymier, L. Janot, J. Li, L. Dobrzynski, Phys. Rev. B 39, 1512 (1989).

[2] J. Friedel, Interaction entre les joints et les impuretés, $4^{e ́ m e}$ Colloque de Métallurgie de Saclay (1960), Métaux et Corrosion $n^{\circ} 428$ (1961). 
[3] S.M. Foiles, Phys. Rev. B 32, 7685 (1985).

[4] R. Riedinger, H. Dreysse, Phys. Rev. B 27, 2073 (1983); ibid. 31, 3398 (1985).

[5] R. Najafabadi, G. Kalongi, Mat. Res. Soc., Symposium Proceeding, Eds. M.H. Yoo, W.A.T. Clarke, C.L. Briant, 122, 275 (1988).

[6] J.R. Smith, J. Ferrante, Phys. Rev. B 34, 2238 (1986).

[7] L. Dobrzynski, Surf. Sci. 180, 505 (1987); Surf. Sci. 200, 435 (1988).

[8] J.P. Muscat, G. Allan, J. Phys. F Metal Phys. 7, 999 (1977).

[9] H.L. Marcus, L.H. Hackett Jr., P.W. Palmberg, Temper Embritllment of Alloy Steels, STP 499, 90, American Society for Testing and Materials, Philadelphia 1972. 